\title{
VARSCOT: variant-aware detection and scoring enables sensitive and personalized off-target detection for CRISPR-Cas9
}

\author{
Laurence O. W. Wilson ${ }^{1 \dagger}$, Sara Hetzel ${ }^{1,2 \dagger}$, Christopher Pockrandt ${ }^{2,3}$, Knut Reinert ${ }^{2,3}$ and Denis C. Bauer ${ }^{1 *}$ (D)
}

\begin{abstract}
Background: Natural variations in a genome can drastically alter the CRISPR-Cas9 off-target landscape by creating or removing sites. Despite the resulting potential side-effects from such unaccounted for sites, current off-target detection pipelines are not equipped to include variant information. To address this, we developed VARiant-aware detection and SCoring of Off-Targets (VARSCOT).

Results: VARSCOT identifies only $0.6 \%$ of off-targets to be common between 4 individual genomes and the reference, with an average of $82 \%$ of off-targets unique to an individual. VARSCOT is the most sensitive detection method for offtargets, finding 40 to $70 \%$ more experimentally verified off-targets compared to other popular software tools and its machine learning model allows for CRISPR-Cas9 concentration aware off-target activity scoring.

Conclusions: VARSCOT allows researchers to take genomic variation into account when designing individual or population-wide targeting strategies. VARSCOT is available from https://github.com/BauerLab/VARSCOT.
\end{abstract}

Keywords: CRISPR-Cas9, Off-target detection, Variants, Genome editing

\section{Background}

The development of the CRISPR-Cas9 system has revolutionized genome-editing [1]. The system can be targeted to almost any genetic sequence through complementary binding to an associated gRNA. Once cleaved, the repair of the break can be manipulated to induce small insertions or deletions or used for the insertion of new sequence $[2,3]$. This has significant implications, particularly in the field of medicine. However, the capacity of CRISPR-Cas9 to bind and cleave at locations other than the target site (termed off-targets), means great care must be taken when using it [4-7]. For this reason, many computational tools have been developed that seek to identify and predict potential offtargets and help inform experimental design [8-10].

Computational detection of off-targets consists of two components: identification and activity prediction. Identification involves identifying alternate sites the CRISPR-Cas9: gRNA complex may bind based on sequence complementarity. In addition to sequence similarity, potential off-

\footnotetext{
*Correspondence: Denis.Bauer@csiro.au

${ }^{\dagger}$ Laurence O. W. Wilson and Sara Hetzel contributed equally to this work.

${ }^{1}$ CSIRO, Sydney, NSW, Australia

Full list of author information is available at the end of the article
}

targets must also be flanked by a Protospacer Adjacent Motif (PAM), a short sequence that the CRISPR-Cas complex must bind to in order to effect cleavage [11]. Detection of potential targets is usually achieved using traditional aligners such as BWA and Bowtie [8]. The likelihood that these target sites could become active off-targets can then be assessed using predictive models. While a number of scoring algorithms are available, the most common models for off-target activity prediction are the Cutting Frequency Determination (CFD) [12], the MIT score for off-target activity [13] and the recently developed Elevation score [9].

Bringing the search and scoring functionality together are pipelines such as CRISPOR [8], which uses BWA to identify potential off-targets and then evaluates them using either the CFD or MIT scores, as well as the Elevation pipeline [9], which uses a custom search tool and model of activity. These pipelines however do have limitations. While read-alignment-based tools offer fast offtarget search, they are limited to very few mismatches between the gRNA and the off-target (typically 5). This is a substantial limitation as highly mutated off-targets with up to 8 mismatches have been recorded in experimental data [7, 14]. Additionally, current pipelines are 
not able to handle variant information. The genetic variations (SNPs, INDELs) found within an individual genome will change the off-target landscape [15-17]. This was recently demonstrated by Lessard et al., who showed experimentally that small variations in an off-target sequence could dramatically alter the cleavage rate of any given site [18]. It is therefore critical that the variant landscape of a genome be taken into account when designing CRISPR-Cas9 gRNAs, particularly for more personalized applications such as gene-therapy [16] and gene-drives [19].

To address both needs we developed VARSCOT (VARiant-aware detection and SCoring of Off-Targets). VARSCOT is able to process variant information provided as a VCF file to identify off-targets that are personalized to an individual. Furthermore, VARSCOT uses a novel seed-and-extend method [20] to allow more mismatches than other alignment-based tools (with a default of 8). VARSCOT also offers a novel machine-learning approach to score off-target activity by taking the sequence composition as well as the relationship between on- and off-targets into account.

Demonstrating the capabilities of VARSCOT, we firstly show how the target-site landscape dramatically changes when taking variant information into account. We then identify features that govern off-target activity and conclude by benchmarking VARSCOT against other activity-predictors as well as the state-of-the-art searchand-scoring pipelines.

\section{Implementation}

\section{Construction of the variant genome}

VARSCOT integrates sequence variants of an individual from a user-defined VCF file by constructing a so-called variant genome that is scanned in addition to the reference genome. The variant genome sequences consist of $22 \mathrm{bp}$ flanking regions upstream and downstream of a given variant that are extracted from the reference genome. For each allele, the corresponding variant is inserted into the sequence. Closely located variants that could potentially be included in a single off-target are extracted and further evaluated within a single sequence. Otherwise off-targets could be included that cannot exist if reference bases are extracted where an individual variant is located.

VARSCOT is intended to be used with phased variants since knowledge of the haplotypes is required in order to extract sequences with multiple variants correctly for each allele. In order to provide a method to process unphased variants, every possible combination of variants for each allele is reported within a sequence.

\section{Read mapping based on Optimum search schemes}

Targets are mapped to the reference and variant genome using a read aligner based on a bidirectional FM index.
As opposed to traditional unidirectional indices, a bidirectional index can search into both directions in any given order thus improving runtime [21]. Most indexbased approximate string matching strategies are still not practical for a large number of errors and already exceed acceptable running times for more than two errors. To allow for up to 8 errors in an index-based search, we use Optimum Search Schemes [20]. This is a strategy that enumerates a pattern with errors in a bidirectional index in such a way, that the number of steps in the index is reduced to a minimum. Using a recent implementation of the bidirectional FM-index based on EPR-dictionaries [22], which is faster by a factor of 2 for DNA alphabets than standard implementations of FMindices based on Wavelet trees, we were able to reduce the search time even further.

After mapping the on-targets back to the reference and variant genome, both results are merged and filtered for the final output. Matches to the reference genome that lie within regions of individual variants need to be filtered out because they do not exist in the present individual and are covered by matches to the variant genome in the same regions. In addition, the original target sites are filtered out since they are always found as perfect matches by the aligner.

For all valid off-targets either the MIT score or Random Forest prediction can be calculated. The resulting off-targets and corresponding scores as well as their positional information and sequence are reported in an output file where the first columns correspond to a BED6 file. Each off-target that contains a variant is tagged as such with a reference back to the input VCF file.

\section{Dataset curation}

We employ two datasets in this study; a Training Dataset (9 on-target and 384 off-targets from [7]) and a Test Dataset (8 on-target and 5314 off-targets from [14]). In these studies, active off-targets were detected using the GUIDE-Seq or SITE-seq methods respectively. For the Training Dataset, we defined active off-targets as any that were detected using the GUIDE-Seq method. We therefore assumed that any sequence with up to $8 \mathrm{mis}$ matches to the on-targets that were not detected were inactive off-targets. Because the number of inactive offtargets was larger than the active class, we performed down sampling. The sampling was weighted based on the mismatch distribution observed in the active offtargets to avoid any imbalances, as there are significantly more inactive off-targets with $>5$ mismatches than in the active class. We repeated the sampling a total of 10 times, creating 10 sets of off-targets to avoid any sampling bias. For the Test Dataset, off-target activity was measured using different concentrations of CRISPRCas9. We defined off-targets as active if they were 
detected at a CRISPR-Cas9 concentration of $64 \mathrm{nM}$, the "standard" concentration used in the original paper.

\section{Model training and feature selection}

A Random Forest classifier was trained using 443 features derived from mismatch properties, sequence context and on-target activity (Additional file 2: Table S1) for each of the active-inactive off-target Training Dataset combinations. Feature importance was extracted for each model and then averaged across all repeats. Afterwards we performed feature selection using a backwards-selection method, where the least important feature is removed and the new model tested. Performance was measured using the out-of-bag error of the model and the combination of features which gave the lowest error were selected as the final model.

\section{Predictive models}

The standalone programs including off-target search for Elevation and CRISPOR were downloaded from their respective repositories. The CFD score was implemented using the scripts from [8] and the MIT off-target score was implemented in a python script using the weights provided in the original paper [13].

VARSCOT and Elevation were run on a 64-bit Linux system with 64 cores and 512 GB RAM. CRISPOR was run on a Macbook Pro with OS X 10.11, two cores and 16 GB RAM.

\section{Results}

\section{VARSCOT identifies unique off-targets using variant information}

To test VARSCOT's ability to predict unique off-targets, we used VARSCOT to compare the predicted off-targets of 100 gRNAs across three individuals of the 1000 genomes project [23]. For this, we limited the prediction of off-targets to sites with up to five mismatches (the maximum number allowed by current state-of-the-art tools) and either the canonical NGG or non-canonical NGA PAM (the most active non-canonical PAM [24]). While the non-canonical NGA PAMA was chosen because it was found to be the most common alternative in experimental datasets [7], VARSCOT also allows users to specify additional non-canonical PAM's to include in the off-target search.

VARSCOT uses a supplied VCF file to generate a "variant genome" which, along with the reference genome, is searched using a seed-and-extend method based on Optimum Search Schemes using bidirectional FM indices [20] for regions similar to a supplied target sequence (Fig. 1a, a more detailed workflow is provided in Additional file 1: Figure S1). This search method allows VARSCOT to identify similar regions with up to 8 mismatches in a $23 \mathrm{bp}$ sequence. Once identified, regions from the variant genome are compared to the reference genome to identify potential off-targets unique to the individual.

As shown in Fig. 1b, only $0.6 \%$ off-targets (134 sites) are consistent between all individuals and the human reference genome (hg19 assembly). In fact, the mutations an individual carries causes on average $98.97 \%$ of the off-targets $(22,570$ sites per genome, $\mathrm{SE}=30)$ to be different when compared to the reference genome. Strikingly, the difference among the individuals is less with $81.68 \%$ of off-targets $(18,626$ sites per genome, $\mathrm{SE}=125)$ unique to an individual. These results showcase the limitations of using a reference genome to identify offtargets for an individual and highlight the importance of understanding an individual's variant landscape.

The relationship between on- and off-target governs activity Variants have the potential to create a significant number of new CRISPR-Cas9 binding sites, however binding does not always translate to cleavage. Similar to on-target activity [25], off-target activity can be predicted based on the sequence of the gRNA and the off-target $[8,9]$.

We trained a Random Forest classifier on a dataset where off-target activity was measured using the GUIDE-Seq method [7] (the Training Dataset) to take the single and di-nucleotide composition of a site as well as the number, position and type of mismatches into account when predicting off-target activity. Here, we chose to focus on off-targets with canonical PAM sequences only, as other PAMs were not well represented in the training set. We also limited the dataset to off-targets with only up to 8 mismatches. While off-targets with more mismatches have been reported, these are typically in ex situ experiments where the genomic DNA has been isolated and treated directly with CRISPR-Cas9 increasing the activity of even heavily mutated sites. In the Training Set, which is an in situ dataset, sites with more than 5 mismatches have a very low activity level hence likely rendering sites with more than 8 mismatches completely inactive (Additional file 1: Figure S2).

As the dataset only contains active off-targets, we assembled a list of inactive sites by randomly sampling the genome, matching the active off-targets in sequence complementarity and number of sites. To avoid selection bias, we repeat the sampling 10 times. For more details, see the methods section.

Using these datasets, we constructed 10 different models of off-target activity (one for each combination of active and inactive targets) and extracted the average feature importance across all models. Consistent with previous reports and as shown in Fig. 1c, features such as the number and position of mismatches were deemed important, particularly if the mismatches fell within the seed-region (the $12 \mathrm{bp}$ immediately upstream of the 
a
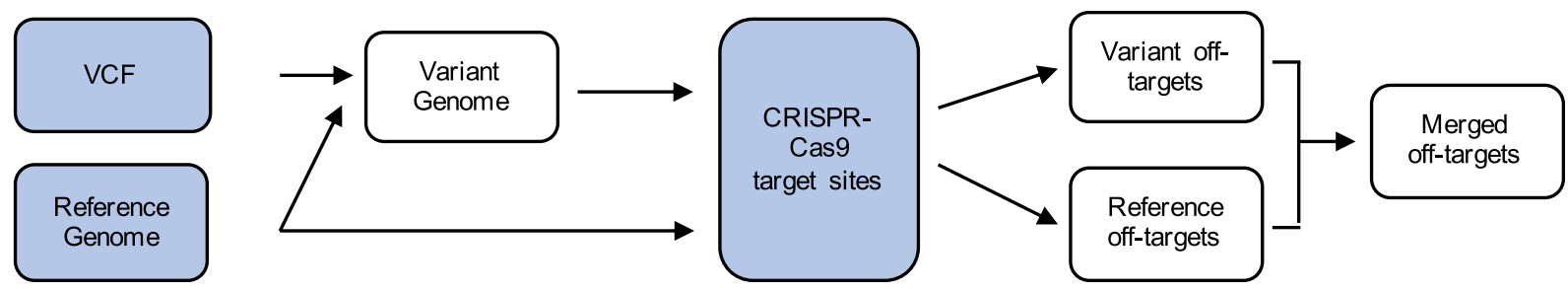

b
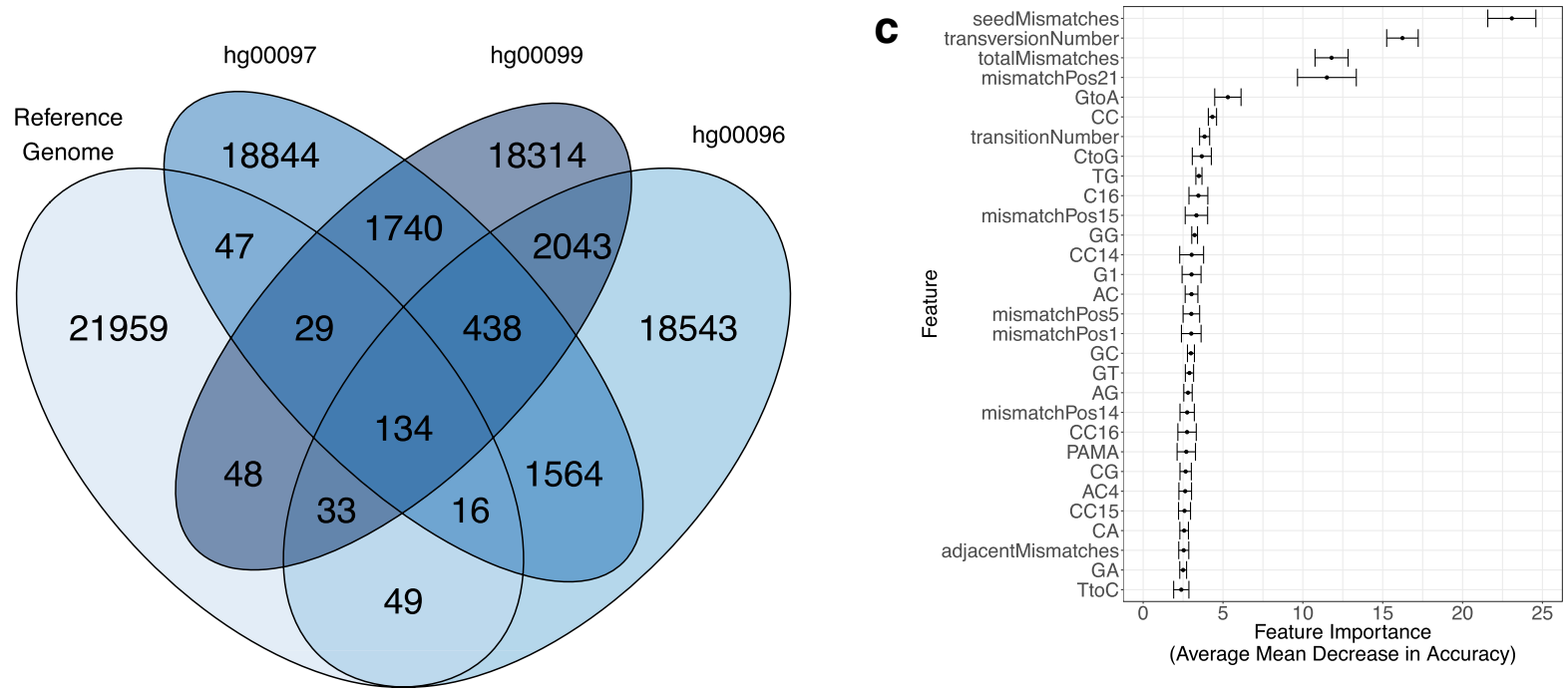

d

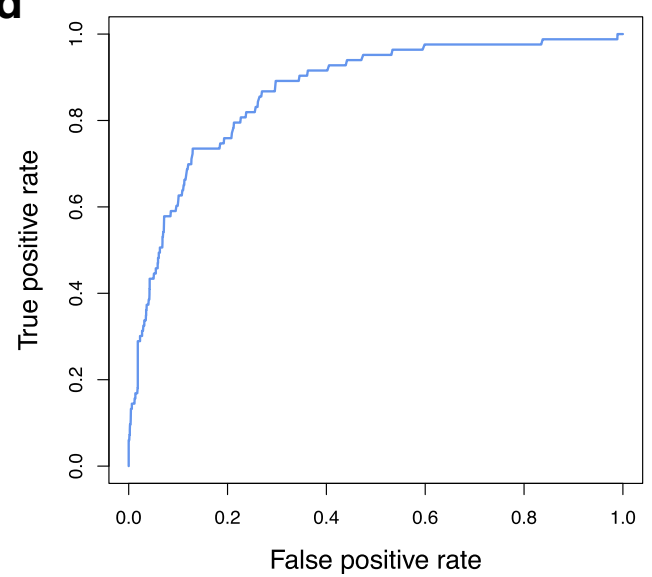

e

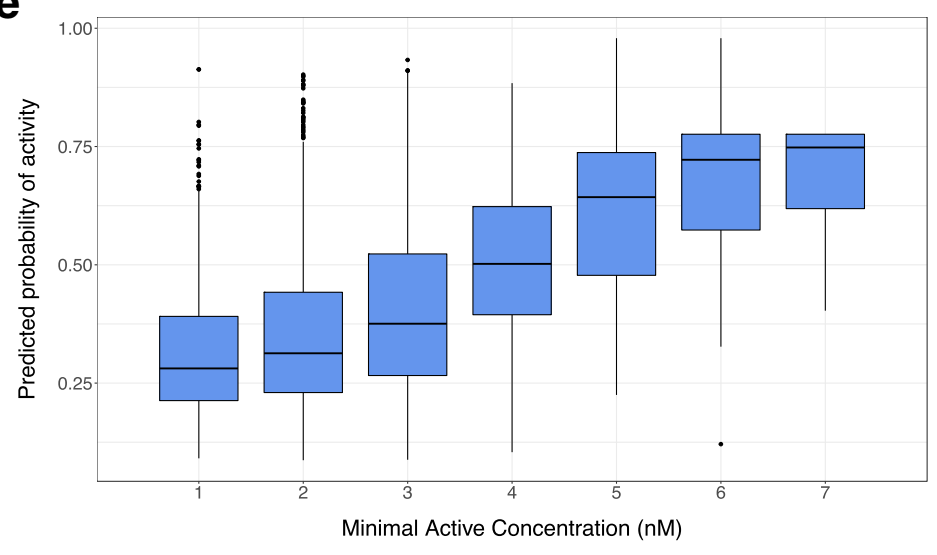

Fig. 1 Development and testing of the VARSCOT model (a) VARSCOT uses a supplied VCF to produce a variant genome which is searched alongside the reference genome to identify variant off-targets. User supplied files are shown in blue, while files generated by VARSCOT are shown in white. $\mathbf{b}$ VARSCOT was used to detect potential off-targets for $100 \mathrm{gRNAs}$ using variant information from three individuals from the 1000 Genomes project. Potential off-targets were compared between individuals and with the hg19 Reference Genome to identify unique targets. c Feature importance for predicting off-target activity. d Receiver Operating Characteristic (ROC) curve of the VARSCOT model tested on the independent Test Dataset filtered for targets with 8 or fewer mis-matches and NGG or NGA PAMs (e) Correlation of the VARSCOT Predicted Probability of Activity with the Minimal Active Concentration of CRISPR-Cas9 used in the Test Dataset

PAM). In addition, it was also found to be important whether the mismatch was caused by a purine/pyrimidine (transversion) or purine/purine or pyrimidine/pyrimidine substitution (transition), suggesting that structural differences between the gRNA and potential off-target influence overall activity.
Interestingly, whether the first base of the PAM at the off-target position matched that at the on-target position, was the 57th most important feature in our model. This position is known to be important for regulating on-target activity of a gRNA [25]. We hence hypothesize that the model uses it to estimate 
on-target activity as an influencing factor on offtarget activity.

In order to identify the minimal number of features required to accurately model off-target activity, we performed feature selection using a backwards-selection strategy, identifying 80 key features. On average a crossvalidated Area Under the Curve (AUC) of 0.956 ( $\mathrm{SE}=$ 0.005) was achieved for training with selected features only and $0.955(\mathrm{SE}=0.006)$ for training with all features (Additional file 1: Figure S3). This suggests that feature selection does not significantly improve performance (paired $t$-test $p=0.41$ ) but rather allows us to exclude unnecessary information. From the 10 training sets, we selected the best-performing model as final model for validation.

\section{Off-target activity can be modelled using only the target sequence}

To confirm the model generalizes after feature-selection and training we validate its performance on an independent Test Dataset by Cameron et al. [14]. This Test Dataset consists of off-targets that were detected across a range of CRISPR-Cas9 concentrations. For this validation test, we considered an off-target active if it was active at a CRISPR-Cas9 concentration of $64 \mathrm{nM}$ (the standard concentration used by Cameron et al.). Testing our model on this dataset yields an AUC of 0.85 (Fig. 1d). This is especially remarkable as our model was trained on off-targets with canonical PAMs (AUC of 0.86 for canonical and 0.83 for non-canonical PAMs, Additional file 1: Figure S4). This indicates that offtarget activity is primarily driven by the target sequence of the off-target and the gRNA and not the PAM.

We also tested if the predicted activity score correlates with the concentration-dependent activity of the off-targets in the Test Dataset. We divided off-target sites in the Test Dataset into groups based on the minimum CRISPR-Cas9 concentration they were active at (with a lower minimum concentration equalling a more active off-target) and plotted the corresponding average predicted activity score from our model. Our results show a clear correlation between activity-score and concentration-score (Fig. 1e), suggesting that our model can also be used to predict activity of offtargets at different CRISPR-Cas9 concentrations.

\section{Comparison with other scores for off-target activity}

We compared our model with the previously published offtarget activity scorers, the MIT [13] and CFD score [12] as well as the Elevation score [9]. These were shown to outperform other available scores in a recent review and therefore represent the currently best scoring schemes [8].

Figure 2a shows the resulting ROC curves on the independent Test Dataset. All models showed strong performance with AUCs $>0.83$. Pairwise comparison showed that only the MIT and Elevation as well as the
MIT and CFD scores were significantly different, with the MIT score outperforming both ( $p$-values $=0.009$ for both comparisons, all other comparisons $p$-value $>0.05$ ). Because there was no significant difference between the performance of our and the MIT model, we elected to include both in the final VARSCOT pipeline.

\section{The VARSCOT pipeline outperforms current off-target detection and activity prediction pipelines}

The performance of VARSCOT was compared to the state-of-the-art off-target detection and activity prediction pipelines Elevation [9] and CRISPOR [8]. VARSCOT was run allowing up to 8 mismatches, while CRISPOR was limited to 5 mismatches and Elevation allowed 6 (up to 3 within the 17 bases proximal to the PAM and any number of mismatches in the three most distal bases) mismatches, respectively. For CRISPOR this was due to the limitations in BWA and for Elevation this was due to runtime as a search with comparable mismatch-number would have taken an order of magnitude longer (hours for a single on-target compared to minutes for both other methods). In order to enable a fair comparison, VARSCOT was used without variant information and Elevation and VARSCOT were limited to canonical NGG and non-canonical NGA PAMs.

As shown in Fig. 2b, of the 4443 sites in the Test Dataset VARSCOT identifies the most out of all methods (1747, $39 \%$ of observed sites), followed by Elevation (1103, 25\%) and CRISPOR (790, 18\%). Of the missed sites, 77\% (2078 sites) were missed due to VARSCOT limiting detection to off-targets with up to 8-mismatches, and the remaining $23 \%$ (618 sites) were missed due to the presence of noncanonical PAMs. Critically, the missed sites were predominantly low-activity off-targets confirming that VARSCOT identifies the active off-targets of interest (Additional file 1: Figure S5).

All three pipelines report more off-targets than are reported in the Test Dataset. VARSCOT identifies an additional 1,354,308 sites, while CRISPOR and Elevation report an additional 14,212 and 23,447 sites respectively. In order to filter out false-positives, a probability cut-off based on our predicted score can be used. Using a cut-off threshold of 0.5 reduces the number of false positives from 1,356,055 to 18,764 (a reduction of approximately $98 \%$ ). While a higher threshold will reduce this further, it also reduces the number of true positives. Care must therefore be taken when choosing a threshold, although it is critical to note that the false positives reduces at a faster rate than the true positives (Fig. 2c). Using a cut-off of 0.4 yields a truepositive rate of $23 \%$ and a false-positive rate of $5 \%$.

\section{Discussion}

VARSCOT is a newly developed off-target detection and scoring tool for CRISPR-Cas9, which incorporates the 


\section{a}

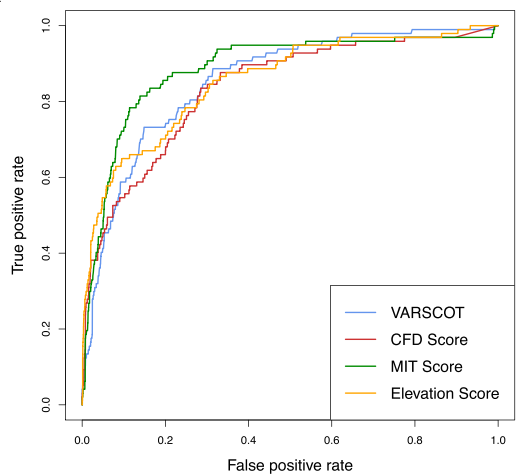

b

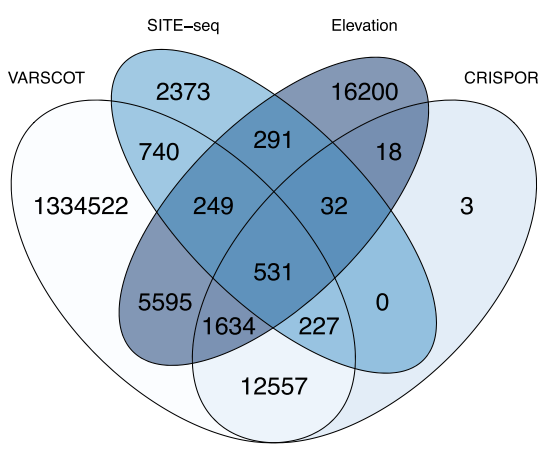

C

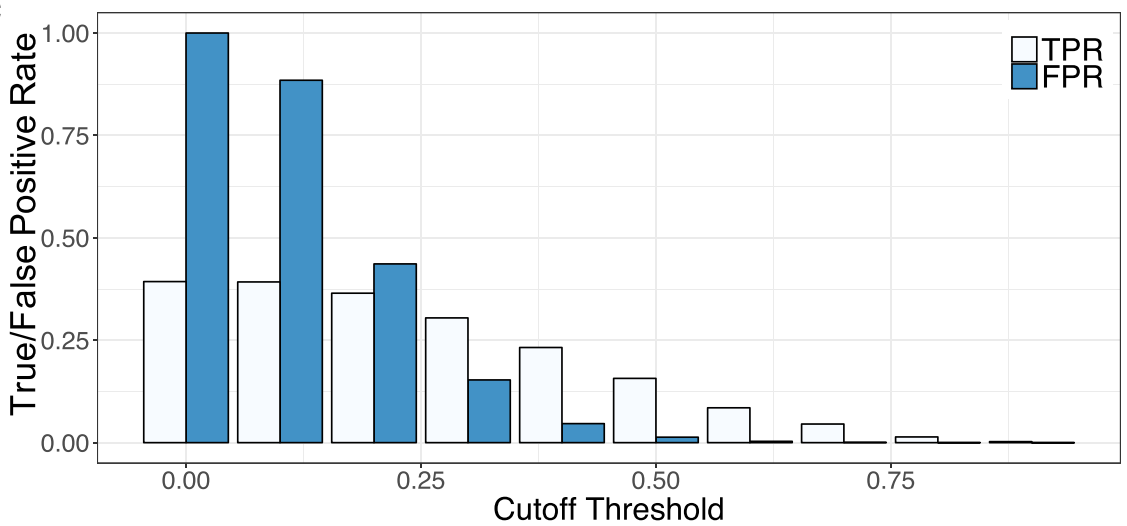

Fig. 2 Comparison of the VARSCOT pipeline (a) Receiver Operating Characteristic (ROC) curves of VARSCOT and other off-target activity models tested on the Test Dataset. $\mathbf{b}$ Number of potential off-targets detected by the VARSCOT, Elevation and CRISPOR pipelines compared to the off-targets detected experimentally in the Test Dataset. $\mathbf{c}$ Effect of a threshold on the True Positive Rate (TPR) and False Positive Rate (FRP) of detection of VARSCOT

variant information of individuals into the search. We have shown that when considering the specific genetic landscape of an individual, 99\% of off-targets are unique and would be missed when scanning a reference genome only. Hence SNP-aware off-target detection is critical for any application of CRISPR which requires an element of personalization, such as genetherapy [16]. VARSCOT is also capable of handling population level variant information. This will be of great use in fields such as gene-drives [19], where individual genome variants about the targeted species cannot be known but population level information on genetic variation at specific loci is available.

VARSCOT detects off-targets in the variant and reference genome using a method based on Optimum Search Schemes using a bidirectional FM index, which is more sensitive and identifies off-targets with more mismatches than traditional aligners. While the default of allowing up to 8 mismatches means VARSCOT captures more validated off-targets than other pipelines, this cutoff can be increased to identify more divergent off-targets. However, this would increase the possibility of false positives which must then be accounted for.
The effect of false positives can be mitigated by using a model to predict the activity of a potential off-target such as the one we developed. While we limited the Training data of our model to only off-targets with NGG or NGA PAMs, critically our model could accurately predict the activity of off-targets with other PAMs (Additional file 1: Figure S4b). In the Test Dataset, applying a standard cutoff of 0.5 reduced the number of false positives by approximately $98 \%$. Deciding on a probability threshold will be a critical step for future experimental design and the correct threshold will depend on the parameters.

Our results showed that the predicted on-target activity of a gRNA is an important factor of off-target activity, suggesting that more care should be taken with gRNAs selected for on-target activity as they will likely have more active off-targets. Similarly, an experiment that uses a higher concentration of CRISPR-Cas9 should be cautious, as previously inactive off-targets could become active.

\section{Conclusions}

Natural genomic variants can have a profound impact on the off-target activity of CRISPR-Cas9 and 
accounting for this variation is therefore critical. VARSCOT is the first off-target detection tool that can account for genetic variation and identify off-targets unique to an individual genome. This will be critical for future work seeking to apply CRISPR-Cas9 to wild type populations or potentially in the clinic.

Availability and requirements Project name: VARSCOT. Project home page: https://github.com/BauerLab/ VARSCOT

Operating system(s): Platform independent.

Programming language: Python and $\mathrm{C}++$.

Other requirements: Python 2.7 with numpy, scipy, sklearn 0.19.0 and pybedtools. R3.4.2 with randomForest. CMake and gcc.

License: CSIRO Non Commercial Source Code License Agreement v1.0.

Any restrictions to use by non-academics: License required for non-academic use.

\section{Additional files}

Additional file 1: Figure S1. Overview of the VARSCOT pipeline. Figure S2. Correlation of activity and mis-match number. Figure S3. Results of Feature Selection. Figure S4. Performance of the VARSCOT model. Figure S5. Distribution of detected and non-detected off-targets. (DOCX 440 kb)

Additional file 2: Table S1. Feature list for predictive model of off-target activity. (DOCX $16 \mathrm{~kb}$ )

\section{Authors' contributions}

S.H. implemented VARSCOT. L.W., S.H. and D.B. conceived of and designed experiments, L.W., S.H. and D.B. analyzed the results. C.P. and K.R. developed the search strategy. L.W., S.H. and D.B. wrote the manuscript. All authors have read and approved the manuscript.

\section{Funding}

This research was supported by The Commonwealth Scientific and Industrial Research Organisation and CSIRO Synthetic Biology Future Science Platform.

Availability of data and materials

All data analyzed here as well as the VARSCOT pipeline are available from https://github.com/BauerLab/VARSCOT

\section{Ethics approval and consent to participate}

Not applicable.

\section{Consent for publication}

Not applicable.

\section{Competing interests}

The authors declare that they have no competing interests.

\section{Author details}

${ }^{1}$ CSIRO, Sydney, NSW, Australia. ${ }^{2}$ Freie Universität, Berlin, Germany. ${ }^{3}$ Max Planck Institute for Molecular Genetics, Berlin, Germany.

Received: 6 August 2018 Accepted: 17 June 2019

Published online: 27 June 2019

\section{References}

1. Doudna JA, Charpentier E. Genome editing. The new frontier of genome engineering with CRISPR-Cas9. Science. 2014;346:1258096.
2. Sawatsubashi S, Joko Y, Fukumoto S, Matsumoto T, Sugano SS. Development of versatile non-homologous end joining-based knock-in module for genome editing. Sci Rep. 2018;8:593.

3. Zaboikin M, Zaboikina T, Freter C, Srinivasakumar N. Non-homologous end joining and homology directed DNA repair frequency of double-stranded breaks introduced by genome editing reagents. PLoS One. 2017;12: e0169931.

4. Kim D, et al. Digenome-seq: genome-wide profiling of CRISPR-Cas9 offtarget effects in human cells. Nat Methods. 2015;12:237-43, 1 p following 243.

5. Kim D, Kim S, Kim S, Park J, Kim J-S. Genome-wide target specificities of CRISPR-Cas9 nucleases revealed by multiplex Digenome-seq. Genome Res. 2016;26:406-15.

6. Frock RL, et al. Genome-wide detection of DNA double-stranded breaks induced by engineered nucleases. Nat Biotechnol. 2015:33:179-86.

7. Tsai SQ, et al. GUIDE-seq enables genome-wide profiling of off-target cleavage by CRISPR-Cas nucleases. Nat Biotechnol. 2015;33:187-97.

8. Haeussler $M$, et al. Evaluation of off-target and on-target scoring algorithms and integration into the guide RNA selection tool CRISPOR. Genome Biol. 2016:17:148.

9. Listgarten J, et al. Prediction of off-target activities for the end-to-end design of CRISPR guide RNAs. Nat Biomed Eng. 2018:2:38-47.

10. Bae S, Park J, Kim J-S. Cas-OFFinder: a fast and versatile algorithm that searches for potential off-target sites of Cas9 RNA-guided endonucleases. Bioinformatics. 2014;30:1473-5.

11. Kleinstiver BP, et al. Broadening the targeting range of Staphylococcus aureus CRISPR-Cas9 by modifying PAM recognition. Nat Biotechnol. 2015;33: 1293-8.

12. Doench JG, et al. Optimized sgRNA design to maximize activity and minimize off-target effects of CRISPR-Cas9. Nat Biotechnol. 2016;34:184-91.

13. Hsu PD, et al. DNA targeting specificity of RNA-guided Cas9 nucleases. Nat Biotechnol. 2013;31:827-32.

14. Cameron P, et al. Mapping the genomic landscape of CRISPR-Cas9 cleavage Nat Methods. 2017;14:600-6.

15. Canver $\mathrm{MC}$, et al. Variant-aware saturating mutagenesis using multiple Cas9 nucleases identifies regulatory elements at trait-associated loci. Nat Genet. 2017:49:625-34

16. Scott DA, Zhang F. Implications of human genetic variation in CRISPR-based therapeutic genome editing. Nat Med. 2017:23:1095-101.

17. Canver MC, Joung JK, Pinello L. Impact of genetic variation on CRISPR-Cas targeting. The CRISPR Journal. 2018;1:159-70.

18. Lessard S, et al. Human genetic variation alters CRISPR-Cas9 on- and offtargeting specificity at therapeutically implicated loci. Proc Natl Acad Sci U S A. 2017:114:E11257-66.

19. Drury DW, Dapper AL, Siniard DJ, Zentner GE, Wade MJ. CRISPR/Cas9 gene drives in genetically variable and nonrandomly mating wild populations. Sci Adv. 2017:3:e1601910.

20. Kianfar, K., Pockrandt, C., Torkamandi, B., Luo, H. \& Reinert, K. FAMOUS: fast approximate string matching using OptimUm search schemes. (2017).

21. Lam, T. W. et al. High throughput short read alignment via bi-directional BWT. In 2009 IEEE International Conference on Bioinformatics and Biomedicine 31-36 (IEEE, 2009). doi:https://doi.org/10.1109/BIBM.2009.42.

22. Pockrandt, C., Ehrhardt, M. \& Reinert, K. in Research in computational molecular biology (ed. Sahinalp, S. C.) 10229, 190-206 (springer international publishing, 2017).

23. 1000 Genomes Project Consortium, et al. A map of human genome variation from population-scale sequencing. Nature. 2010;467:1061-73.

24. Zhang Y, et al. Comparison of non-canonical PAMs for CRISPR/Cas9mediated DNA cleavage in human cells. Sci Rep. 2014:4:5405.

25. Wilson LOW, Reti D, O'Brien AR, Dunne RA, Bauer DC. High activity targetsite identification using phenotypic independent CRISPR-Cas9 Core functionality. CRISPR J. 2018;1:182-90.

\section{Publisher's Note}

Springer Nature remains neutral with regard to jurisdictional claims in published maps and institutional affiliations. 